\title{
Bit level Systolic Implementation on LMS adaptive filter
}

\author{
T. Honjo, K. Tanaka and T. Nakamizo \\ Dept. of Computer Science, The National Defense Academy
}

\begin{abstract}
Although LMS adaptive filters have been applied to many signal processing applications, there exis ts a critical limitation of the conventional adaptive filter when it is applied for a real time signal processing. It is simply because of its required lengthy convolution (filtering) calculation. To overcome the limitation, we propose a new Delayed LMS algorithm which is implemented in Signed-Digit Number sys tem. In the paper, a new pipelined adaptive filter is developed and its detailed analysis is described with an actual VLSI implementation.
\end{abstract}

\section{Introduction}

Adaptive digital filters have been applied to wide range of applications, such as channel equalization, noise cancellation or echo cancellation. Many adaptation algorithms including least mean square (LMS), recursive least square (RLS) and adaptive lattice algorithm, are frequently us ed for updating filter weights. Among these adaptive algorithms, because of its relative computational simplicity and easiness to be implemented on a VLSI circuit, the LMS algorithm has been paid a great deal of attention and successfully applied in numerous applications. Since the recent emerging VLSI technology can be effectively used to integrate the complex system on a silicon, adaptive filters using VLSI technology is highly demanding area in the field. In general, adaptive filter consist of two sections, the filtering section for computing the filter output and the adaptation section for updating the filter weight. The error from filtering section, which is the computational burden in LMS adaptive FIR filter, must be used to update filter weight before a next sample arrives. Because of this, in some practical applications for high speed real time signal processing there is a critical limitation on its implementation. The methods to overcome this problem can be considered as follows [1].

(1) Pipelining and parallel processing

(2) Implementation of high-speed mathematical operator.

Because of the computational delay between the filtering section and the adaptation section, it is impossible to use pipelining method for conventional LMS algorithm. However, it has been shown recently that it is possible to pipeline by introducing delayed least mean square (DLMS) algorithm, which uses delayed error to update the filter weights instead of using the current error. In 
our adaptive method, we implemented th is DLMS algorithm to realize a perfect pipelining of adaptive filters. With regard to the method (2), a conventional method (binary number system) has been reported in the past. However, in binary system there is a long carry propagation delay. In the paper, the accumulator using Signed Digit Number Representation (SDNR) which allows computation to be carried out without a long propagation delay is implemented. To realize a high speed pipelined parallel adaptive filter, a new method of implementing SDNR on DLMS algorithm is proposed in the paper.

\section{Signed Digit Number Representation}

SDNR is considered to be a class of number representation system. This number system was introduced by Avizienis [2] in 1961 to realize a fast computation by reducing a long carry propergation in mathematical operation (addition, subtraction or multiplication, division). As compared against conventional number representation, signed digit number representation has additional several sign digits depending on the selection of a radix number size. Since th is causes an increase of system's hardware requirement, it had been difficult to implement on a circuit. However it is now possible to implement SDNR because of the recent development of VLSI integration technology. In general, a number can be expressed in the following equation.

$$
Z=\sum_{i=-n}^{m} z_{i} r^{-i}
$$

In equation (1), $\mathrm{r}$ is a radix and $\mathrm{zi}$ can be defined as $\{-(r-1) \ldots 0 \ldots r-1\}$. There are following differences between SDNR and conventional number system:

(1) Because zi may assume negative as well as positive values, there is no sign at the
Most Significant Digit (MSD).

(2) There may be several possible representation for any given value.

The redundancy in SDNR allows a method of mathematical operation, in which each accumulation result is the function of the digits in two or three adjacent positions of the operands. The mathematical operator has the following important properties.

(1) It allows mathematical operations to be carried out without a long carry propagation from the least significant digit to the most significant digit.

(2) The time needed for the operation is constant and does not depend on the word length.

(3) The operation generates its result the most significant digit first .

For example, we consider the addition of SD number $\mathrm{Z}_{\mathrm{i}}$ and $\mathrm{Y}_{\mathrm{i}}$. This operation can be done by two steps as following.

$$
\begin{aligned}
& Z_{i}+Y_{i}=r t_{i-1}+W_{i} \\
& S_{i}=W_{i}+t_{i}
\end{aligned}
$$

$\mathrm{S}_{\mathrm{i}}$ is a sum digit, $\mathrm{W}_{\mathrm{i}}$ is an interim sum digit and $t_{i-1}$ is a transfer digit. The block diagram of equation (2) and (3) are shown in Fig.1. In block of type (3), because transfer digit $t_{i}$ is generated to satisfy the range of values which $S_{i}$ as sumes in a radix number size, each accumulation result is the function of the digit in two adjacent positions of the operand.

\section{DLMS filter [3]}

In the paper, adaptive filter is implemented as a linear prediction filter. In the linear prediction theory, the filter output is a predicted estimate and can be represented as follows 


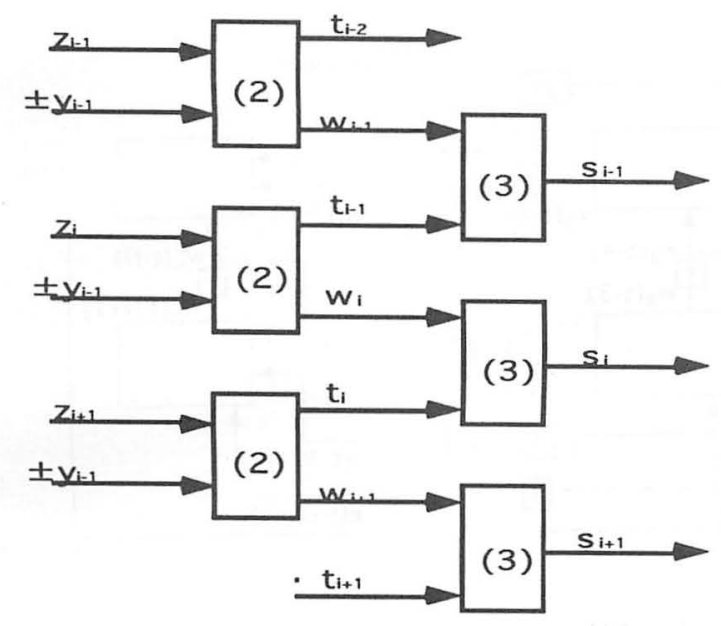

Fig. 1. The block diagram of adder

$$
\widehat{x}(n)=\sum_{k=1}^{p} C_{k} x(n-k)
$$

In the above equation, $x(n)$ is a predicted value of $x(n)$ based on the data up to time $n-1$, the $C_{k}$ is the filter weight, and $\mathrm{p}$ is the order of the filter.

The DLMS algorithm with respect to equation (4) is represented as follows:

$$
\begin{gathered}
\mathbf{W}(t)=\mathbf{W}(t-1)+\beta e(t-D) \mathbf{X}(t-D) \\
e(t)=x(t)-\hat{x}(t) \\
\hat{x}(t)=\mathbf{X}^{T}(t) \mathbf{W}(t-1) \\
\mathbf{W}(t)=\left[w_{1}(t), w_{2}(t), \ldots \ldots, w_{N}(t)\right]^{T} \\
\mathbf{X}(t)=[x(t-1), x(t-2), \ldots \ldots, x(t-N)]^{T}
\end{gathered}
$$

In equation (5)-(7), $W(t)$ is a weight vector and $X$ $(t)$ is a input vector. $\beta$ is a step size used for adaptation of the weight vector. $\mathrm{D}$ is the delay and $\mathrm{N}$ is the order of the filter. In the case when $\mathrm{D}=0$, equation (5)-(7) are equal to conventional filtering.

In general, basic type of pipelining for high speed real time signal proces sing application can be implemented in a structure shown in Fig. 2.

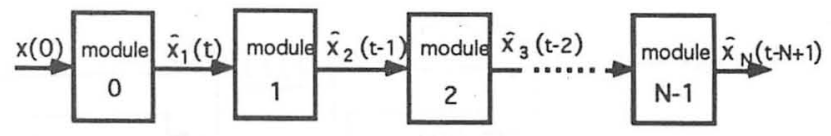

Fig.2 Basic type of pipelinig

Equations (5)-(7) are remapped on to $\mathrm{N}$ identical linear array like Fig. 2 by a perfect pipelining. This implementation will introduce $\mathrm{N}$ adaptive delays. In general the delay $\mathrm{D}$ is selected as equal to $\mathrm{N}$. The ou tput vector of each array is repres ented as

$$
\widehat{\mathbf{X}}(t)=\left[\widehat{x}_{1}(t), \hat{x}_{2}(t-1), \ldots \ldots, \widehat{x}_{N}(t-N+1)\right]^{T}
$$

The data flows of each data is indicated in Fig. 2. In dividual element of the predicted vector satis fies the following relation ship.

$$
\begin{aligned}
\hat{x}_{i+1}(t-i)=\sum_{k=1}^{i+1} x(t-i-k) w_{k}(t-i-1) \\
=\left(\sum_{k=1}^{i} x([t-1]-[i-1]-k)\right. \\
\left.\quad \times w_{k}([t-1]-[i-1]-1)\right) \\
\quad+x(t-2 i-1) w_{i+1}(t-i-1) \\
=\hat{x}_{i}(t-i)+x(t-2 i-1) w_{i+1}(t-i-1)
\end{aligned}
$$

Considering this implementation of these identical liner arrays, $i$ th weight $\mathrm{w}_{\mathrm{i}}(\mathrm{t}-\mathrm{i}-1)$ must be updated at the same module. From (5) and (8), the update equation for the $i$ th weight can be represented as

$$
w_{i+1}(t-i)=w_{i+1}(t-i-1)+\beta e(t-N-i) x(t-N-2 i-1)
$$

$\mathrm{N}$ pipelined liner array can be implemented by (8) and (9). Fig. 3 shows a construction of pipelined DLMS filter, where a dotted line represent one module. Synchronization between each module must be done by setting appropriate delays on input, output and error signal. Upper part is a filtering section, and lower part is a weight update section. Note that final 


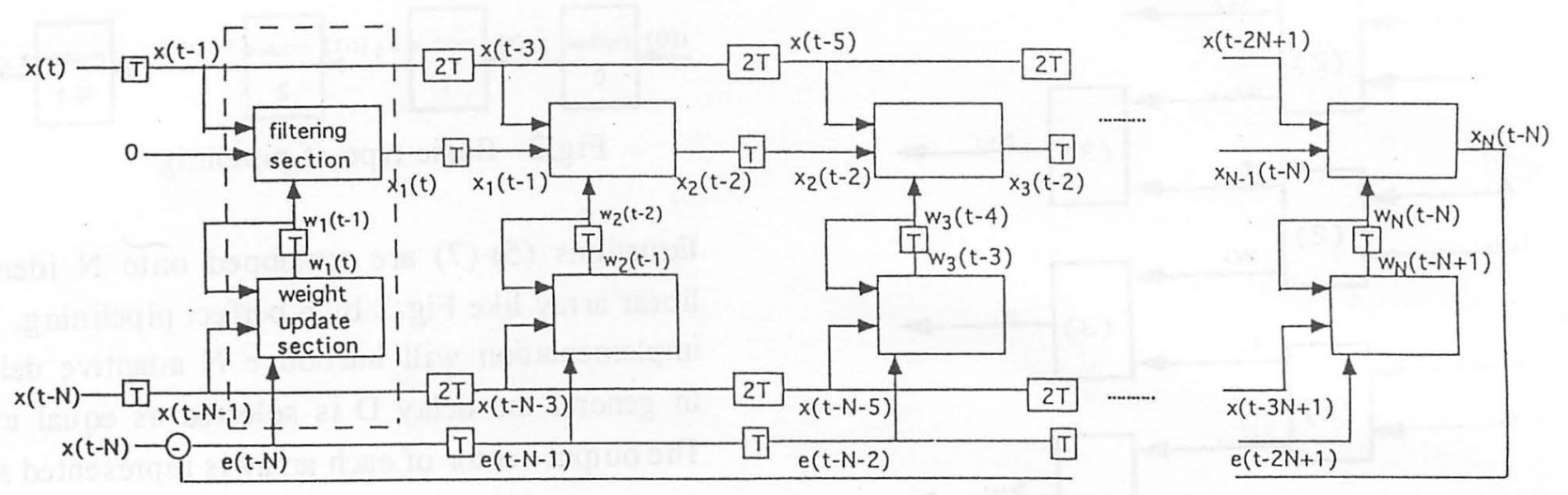

Fig.3 Structure of DLMS adaptive filter

output $x_{n}(t-N)$ is a delayed version of the conventional LMS filter output. With regard to the effect of the delay $D$ for convergence, detailed an alys is using conventional stochas tic method has been reported in [4]. Increas ing the delay D causes more required iterations for convergence. Fig. 4 shows the estimation error of DLMS and that of LMS adaptive filter. The solid line follows an estimation error of DLMS and the dotted line follows the estimation error of LMS. In the begining, convergence of DLMS algorithm is slow, however, tow ards the end it converges as similar to the conventional LMS.

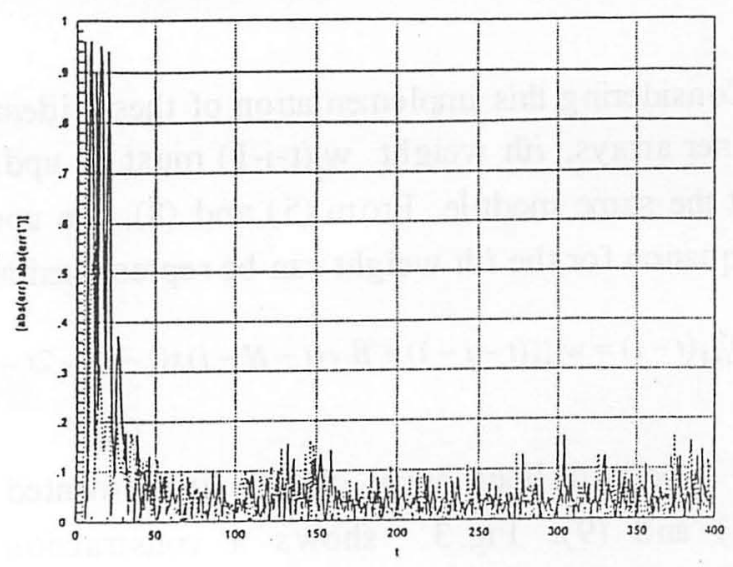

Fig. 4 Estimation error of adaptive filter

In this way both filter output and weight can be updated identically at each module. Thus the ad aptive filter has the following characteristic.
(1) No increase in hardware is needed when the order of the filter is increased.

(2) Because of its modularity, the filter can be ef fectively designed

(3) The required time for each operation is constant and is independent of the filter order.

\section{High speed accumulator [5]}

The block diagram of a pipelined DLMS adaptive filter that executes (8) and (9) are shown in Fig. 3. All computation of the DLMS adaptive filter can be operated by combining of multiplication and addition. The filter section and weight update section in Fig. 3 can be computed by same type of accumulator. To realize a high speed real time operation, multiplier and accumulator are designed using radix-2 SD number implementation which are described in section 2. The number system of our adaptive filter uses the following set of numbers: $\{-1,0,1\}$. However, in actual implementation, single data wire can not represent -1 , we use two data wires, $\mathrm{H}$ and $\mathrm{L}$, which can be used to represent a set of numbers: $\{-1,0,1\}$. Representation methods of radix-2 SD number are indicated in Table.1.

The accumulator unit using above design method is shown in Fig. 5. In this case, both filtering and weight updating can be done on the same unit. 


\begin{tabular}{|c|c|c|}
\hline SD number & H & L \\
\hline 1 & 1 & 0 \\
\hline 0 & 0 & 0 \\
\hline-1 & 1 & 1 \\
\hline
\end{tabular}

Table.1. Representation method

Fig. 5 can be also used for weight updating unit. The processor does one multiplication operation as well as one operation of addition. The in put signal $\beta \mathrm{x}$ is a product of $\mathrm{x}$ and $\beta$ which is computed before signal is inputted. Also the input is in parallel and suitable for either SDNR or 2'scomplement form. The signal $\beta \mathrm{x}$ in the paper is chosen to be in 2's-complement form. The weights and errors are in MSD first format. The subscript of the input name means the order of the bit and the solid black dots indicates a latch to synchronize signals in parallel. The square processor labeled $\mathrm{M}$ is a multiplier, the circle labeled + is a carry save adder and the circle labeled a, b and c, d are adders, whose functions indicated in Fig. 6.

Next, the signal flowgraph of the filter section and weight update section using above accumulators are shown in Fig.7. The dotted line stream shows MSB first signal format and the solid line shows parallel bus format. Throughput of the filter is found to be 9 clocks, which is independent from the word length.

Considering the output conversion from SD

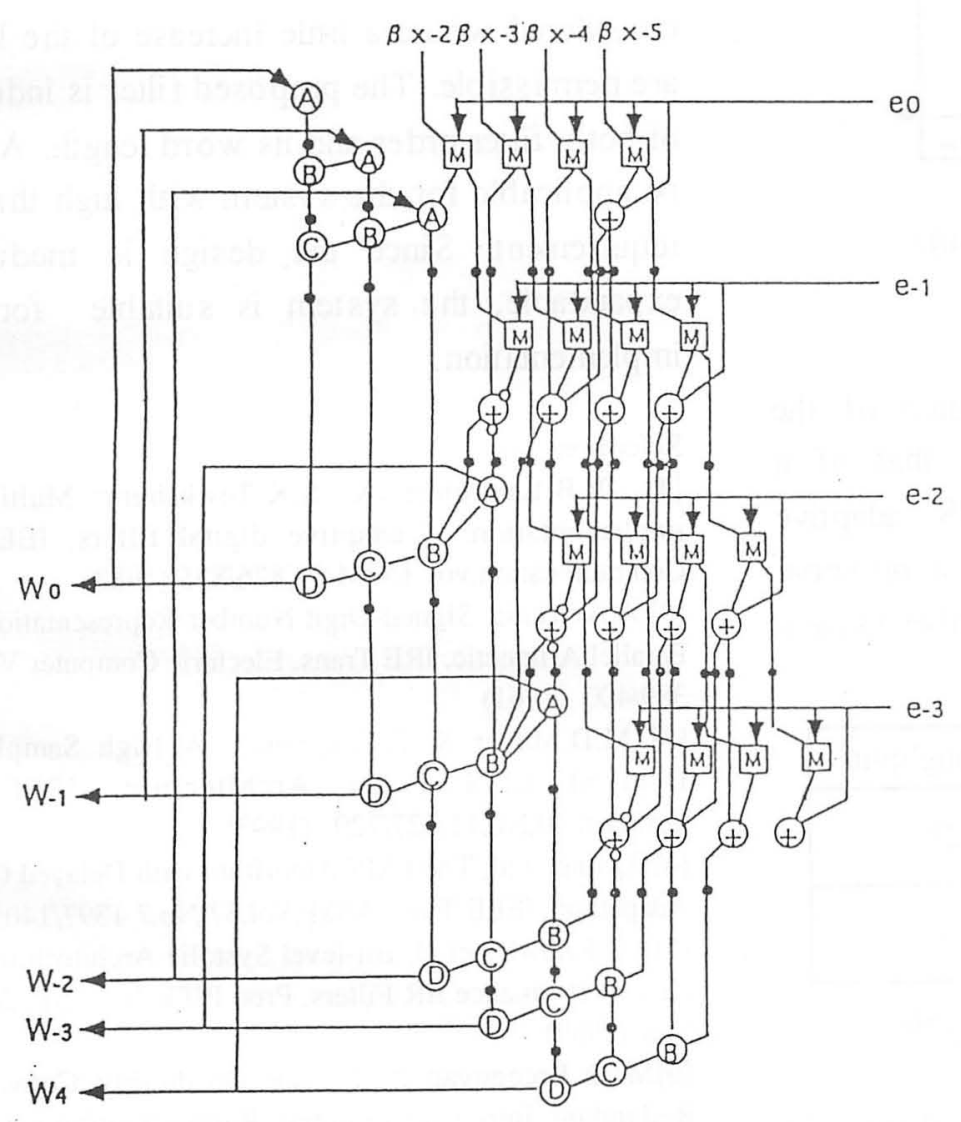

Fig.5 The accumulator based on SDNR
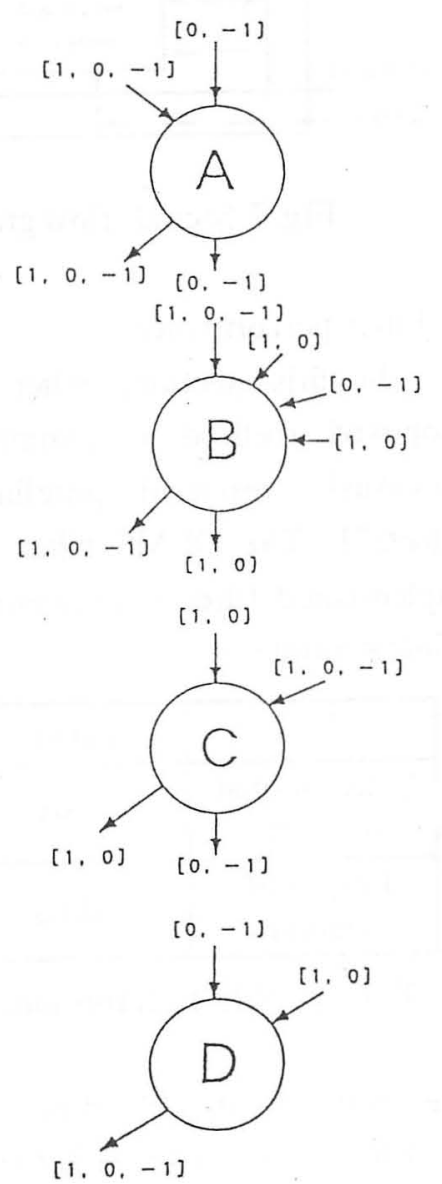

Fig. 6 The functiotions of adders 
number to binary number, there has been a report of on-line algorithm[6] which can be implemented by using a piplined circuit[5]. To make use of the designed circuit, our conversion circuit is obtained by a slight modification of the circuit in Fig.5. Because a part using carry save adder in Fig. 5 is similar to conventional multiplier and adder unit, converted output can be generated in parallel at the bottom of Fig.5. Since this conversion will not degrade the filter operation, its pipelined form will have no effect on the throughput rate.

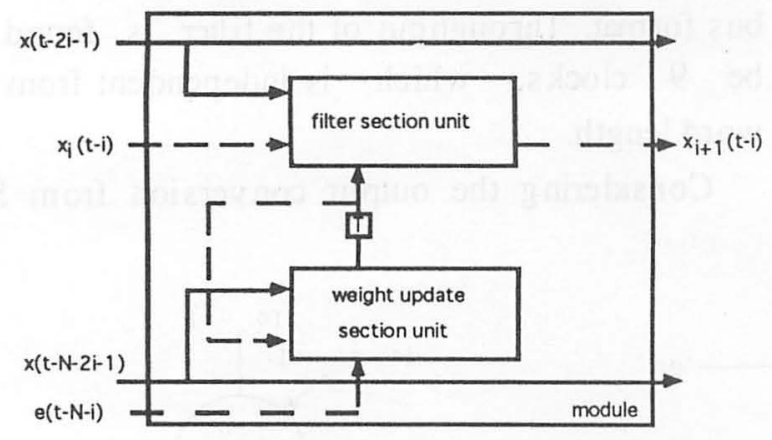

Fig.7 Signal flow grah of module

\section{Filter performance}

In this section, filter performance of the proposed method is compared with that of a previously reported pipelined DLMS adaptive filters[7]. The DLMS filter in [7] is a bit-serial implemented filter in conventional number system (binary number).

\begin{tabular}{|c|c|c|}
\hline & Latency & Throughput \\
\hline $\begin{array}{c}\text { Conventional } \\
\text { method }\end{array}$ & $3 \mathrm{~B}$ & $2 \mathrm{~B}$ \\
\hline $\begin{array}{c}\text { Proposed } \\
\text { method }\end{array}$ & $\mathrm{B}+6$ & 9 \\
\hline
\end{tabular}

Table.2. Filter performance (clock cycle)

The comparison (latency and throughput) of the filters are shown in Table.2. One clock time of the conventional method is equal to three EXOR gates and that of the proposed method is equal to four EX OR gates. B is a w ord length of the filter input signal.

\section{6. summary}

In this paper, a new LMS adaptive filter for high speed real time signal processing application is proposed. The pricipal operation of the filter is designed around DLMS algorithm and its piplined implementation consists of accumulators based on SDNR. DLMS algorithm enabled us to realize a perfect pipelining which was considered to be impossible by the conventional LMS algorithm. As compared against conventional number representation, SDNR has additional hardware depending on the selection of a radix number. However, high computational speed gained by SDNR implementation, which avoids a long carry propagation, could be a critical factor to be considered where a little increase of the hardware are permissible. The proposed filter is independent of both filter order and its word length. And it can be applicable for the system with high throughput requirement. Since the design is modular and expandable, the system is suitable for VLSI implementation.

\section{References}

[1] V.B.Lawrence \& S.K.Tewksbury: Multiprocessor implementation of adaptive digital filters, IEEE Trans. Communication,vol. COM-31,826/835 (1983)

[2] A.Avizenis: Signed-Digit Number Representation for Fast Parallel Arihmetic, IRE Trans. Electoric Computer Vol EC-10, 389/400 (1961)

[3] M.D.Mayer \& D.P.Agrawel: A High Sampling Rate Delayed LMS Filter Architecture, IEEE Trans. CAS,vol.40,No.11,727/729 (1993)

[4]G.Long,et al, The LMS Algorithm with Delayed Coefficent Adaptation, IEEE Tans ASSP,Vol.37,No.7 1397/1405 (1989)

[5]S.C Knowles et al, Bit-level Systolic Architecture for Very High Performance IIR Filters, Proc IEEE ICASSP, 2449/2452 May (1989)

[6]M D Ercegovac \& T Lang:On-the-Fly Conversion of Redundant into Conventional Representation,IEEE Trans Computer,Vol C-37,No7,895/897, (1987)

[7]Chin-Liang Wang: Bit-Serial VLSI Implementation of Delayed LMS Adaptive FIR Filters, IEEE Trans SP,Vol.42,No.8,2169/2175 (1994) 